\title{
Paeoniflorin prevents postoperative formation in an experimental rat model
}

Research Paper

\author{
Qi Gao ${ }^{1}$, Guangbing $\mathrm{Wei}^{1}$, Yunhua Wu${ }^{1}$, Na Yao ${ }^{2}$, Cancan Zhou ${ }^{3}$, Kai Wang ${ }^{1}$, Kang \\ Wang $^{1}$, Xuejun Sun ${ }^{1}$ and Xuqi Li ${ }^{1}$ \\ ${ }^{1}$ Department of General Surgery, The First Affiliated Hospital of Xi'an Jiaotong University, Xi'an 710061, Shaanxi, China \\ ${ }^{2}$ College of Nursing, Shaanxi University of Chinese Medicine, Xianyang 712046, Shaanxi, China \\ ${ }^{3}$ Department of Hepatobiliary Surgery, The First Affiliated Hospital of Xi'an Jiaotong University, Xi'an 710061, Shaanxi, China \\ Correspondence to: Xuqi Li, email: lixuqi@163.com \\ Keywords: peritoneal adhesion, oxidative stress, inflammation, paeoniflorin, postoperation \\ Received: August 14, $2017 \quad$ Accepted: August 28, $2017 \quad$ Published: September 28, 2017 \\ Copyright: Gao et al. This is an open-access article distributed under the terms of the Creative Commons Attribution License 3.0 \\ (CC BY 3.0), which permits unrestricted use, distribution, and reproduction in any medium, provided the original author and source \\ are credited.
}

\section{ABSTRACT}

Although materials and modern surgical techniques have been developed to suppress postoperative adhesions, adhesion formation can still occur, and thus, a novel effective anti-adhesion drug is greatly needed. In the present study, we explored the efficacy of paeoniflorin treatment against postoperative peritoneal adhesions and examined the anti-oxidative stress and anti-inflammatory properties of PE. Forty-eight male Sprague-Dawley rats were randomly divided into 6 groups for the study: the sham, control, hyaluronan and three concentrations (10, 20 and $40 \mathrm{mg} / \mathrm{kg} / \mathrm{d}$ ) paeoniflorin groups. Abdominal adhesions were created by abrasion of the caecum and its opposite abdominal wall. In the paeoniflorin groups, the rats were administered daily oral doses of paeoniflorin for $\mathbf{7}$ days. The abdominal cavities of the rats were reopened with a U-shaped incision to macroscopically grade the adhesions. Histologic analysis was performed, and oxidative stress, inflammatory cytokine, collagen fiber degradation and cytokeratin levels were measured. Macroscopic and histopathological measurements revealed that paeoniflorin reduced peritoneal adhesion and inflammation. Notably, treatment with paeoniflorin reduced the protein levels of TGF- $\beta 1$, IL- 6 and COX-2. The collagen fiber fractions were distinctly lower in the PE groups than in the control group. Western blotting analyses showed that paeoniflorin increased MMP-9 and superoxide dismutase- 2 protein expression and sharply reduced a-SMA and COX-2 protein expression. Peritoneal mesothelium cells were more continuous and complete in animals treated with paeoniflorin. Our study suggests that paeoniflorin can be used to ameliorate peritoneal adhesions via antioxidative stress and anti-inflammatory actions during the postoperative period.

\section{INTRODUCTION}

The incidence of adhesion formation is greater than $93 \%$ after an abdominal surgical procedure $[1,2]$. However, few clinical doctors focus on the high incidence of peritoneal adhesion formation $[3,4]$ or complications, including bowel obstruction, infertility, pelvic pain and other serious conditions [5, 6]. Multiple products for the prevention of peritoneal adhesions have been marketed
[7-10], including barrier materials. The prophylactic effects of these products are limited, and no intervention has been accepted as a criterion in the clinic [11-13].

Peritoneal adhesion is triggered immediately after the peritoneum undergoes surgery, trauma, infection or oxidative stress [14]. Trauma, surgery and injury induce peritoneal mesothelial cell dysfunction and result in fibrinogen-rich severe extravasation, which is then degraded via the process of fibrinolysis. When fibrinolysis 
is insufficient in the damaged peritoneum, adhesion formation develops due to the incomplete resorption of fibrinous deposits. Furthermore, an inflammatory response is also the result of peritoneal adhesion, in addition to dysregulated fibrinolysis and collagen production [15, 16]. Studies $[17,18]$ in rat models have shown that the infiltration of macrophages aggravates adhesion formation by the systematic depletion of macrophages themselves.

Paeoniflorin (PE) is the main active compound in peonies, which are used in traditional Chinese medicine [19]. The pharmacological effects of PE have been demonstrated in previous investigations. In rat models of rheumatoid arthritis [20], PE has been shown to play numerous roles, including the mitigation of oxygenradical damage, anti-inflammatory actions and the elimination of neurotoxicity. Experimental rat models [20] of periodontitis have indicated that PE exerts its effects by decreasing the levels of inflammatory cytokines, including tumor necrosis factor- $\alpha$ (TNF- $\alpha)$, interleukin- $\beta 1$ (IL- $\beta 1$ ) and interleukin-6 (IL-6), by down-regulating the expression of cyclooxygenase-2 (COX-2), metal matrix proteinase-9 (MMP-9) and inducible nitric oxide synthase (iNOS) proteins [21]. In addition, PE significantly decreases the formation of intracellular reactive oxygen species (ROS) and increases the levels of superoxide dismutase (SOD), glutathione (GSH) and endogenous anti-oxidants at a cellular level [22].

Nonetheless, research on role of PE in the prevention of peritoneum adhesion formation has not yet been reported. The objective of the present research was to study the effectiveness of PE, a compound that has been shown to have anti-inflammatory and antioxidative effects, in the prevention of peritoneum adhesion formation in a rat model.

\section{RESULTS}

\section{PE significantly reduces macroscopic peritoneal adhesion scores in a rat model}

All the rats survived to 7 days after the operation, and no significant differences were observed in the body weights of the rats among the six groups (Supplementary Figure 1). The adhesion results are shown in Figure 1A, 1B. The extent and severity of adhesion in the PE groups were less than those in the control group $(P<0.05)$ (Supplementary Tables 1 and 2). The adhesion scores of the M-PE and H-PE groups were significantly lower than those of the control group $(P<0.05)$ (Figure 1C).

The degree of peritoneal adhesion in each group was lower than that in the control group (Figure 1A). In the sham group, there was only a small amount of adhesion near the peritoneal incision, and intestine-to-abdominalwall adhesions were relatively uncommon. In contrast, the control group exhibited numerous omentum-to-bowel, bowel-to-parietal peritoneum, parietal peritoneum-to- omentum, and bowel-to-bowel adhesions. The three groups of rats that had been treated with PE had fewer peritoneal adhesions than the control group. In the animals of the M-PE and H-PE groups, there was a low degree of adhesion formation, which appeared loose, and there was thin adhesion around the incision. The degree of adhesion in the HA group was better than that in the control group.

\section{$P E$ reduces inflammatory cell infiltration, per histopathological examinations}

The histopathological examinations revealed that PE treatment reduced microscopic changes (Figure 2A), such as the increase in inflammatory cells as a result of postoperative peritoneal adhesion, and caused a notable decrease in the histopathological scores relative to the control groups $(P<0.05)$ (Figure 2B). The histopathological scores of the three PE groups were different. The results revealed that the degree of inflammatory cell infiltration and the scores was associated with drug dose. Relative to the PE group, more inflammatory cell infiltration, granulation formation, and increased collagen deposition on the damaged parietal peritoneum and cecal specimens were observed in the control group. The H-PE group contained the fewest number of adhesions and had an optimal score $(P<0.001)$.

\section{PE decreases inflammatory reaction and oxidative stress reaction}

Relative to the control, PE reduced serum inflammatory cytokine levels. TGF- $\beta 1$ and IL- 6 were decreased in the M-PE and H-PE groups $(P<0.05)$ (Figure $3 \mathrm{~A}, 3 \mathrm{~B})$. In the enterocoelic fluid samples, the SOD in the H-PE group and ROS in both the M-PE and H-PE groups were reduced $(P<0.05)$ (Figure 3C, 3D). Western blotting analysis revealed that the expression of COX-2 in rat peritoneal adhesion tissue was decreased after treatment with the PE. The M-PE and H-PE groups exhibited large decreases relative to the control and HA groups (Figure 4). The oxidative stress marker SOD-2 was increased in tissue specimens from the PE treatment groups. The results from the parallel testing of SOD-2 content in our specimens were consistent with those from immunohistochemical staining (Figure 5A, 5D) and western blotting (Figure 4) analyses in the PE groups.

\section{PE inhibits fiber development in the damaged peritoneum and/or adhesion tissues}

When the $\alpha$-SMA concentration was detected via immunohistochemical staining, a significant difference was observed between the assay groups and the control group (Figure 5B, 5E). In the control group, an abundance of spindle fibroblasts with positive brown staining was easily observed in the thick adhesive tissue. However, 
the sham group exhibited little or no positive staining. In the HA and L-PE groups, the number of fibroblasts with positive $\alpha$-SMA expression was slightly lower than the number in the control group. Nevertheless, in the M-PE and H-PE groups, the prevalence of fibers was significantly decreased in the adhesion tissues and/ or damaged peritoneum. The expression of $\alpha$-SMA as detected by western blotting was also significantly different (Figure 4). Furthermore, we evaluated collagen I expression by immunohistochemical staining, which revealed that the deposition transitioned from high to low with the increase in PE doses (Figure 5C, 5F). Collagen I deposition in the HA group was the same as in the L-PE group. Therefore, our research shows that $\mathrm{PE}$ can inhibit the fibrosis of the damaged or adhesive peritoneum with increasing drug concentration in a rat model.

\section{Administration of PE decreases collagen exposition in the impaired peritoneum}

Collagen fibers were stained red via picrosirius red staining (Figure 6A). There was no collagen fiber deposition in the sham group. However, in the control group, hyperplastic collagen fibers were easily detected. The amount of collagen deposition detectably decreased in the adhesion tissue in the PE groups. A minimal amount of collagen deposition and a relatively intact peritoneal architecture were detected in the H-PE group. Collagen deposition in the L-PE group was less than that in the HA group. Furthermore, the adhesions thickness results were equivalent (Figure 6B).

To further understand the degree of the degradation of the extracellular matrix, we evaluated MMP-9

\section{A}
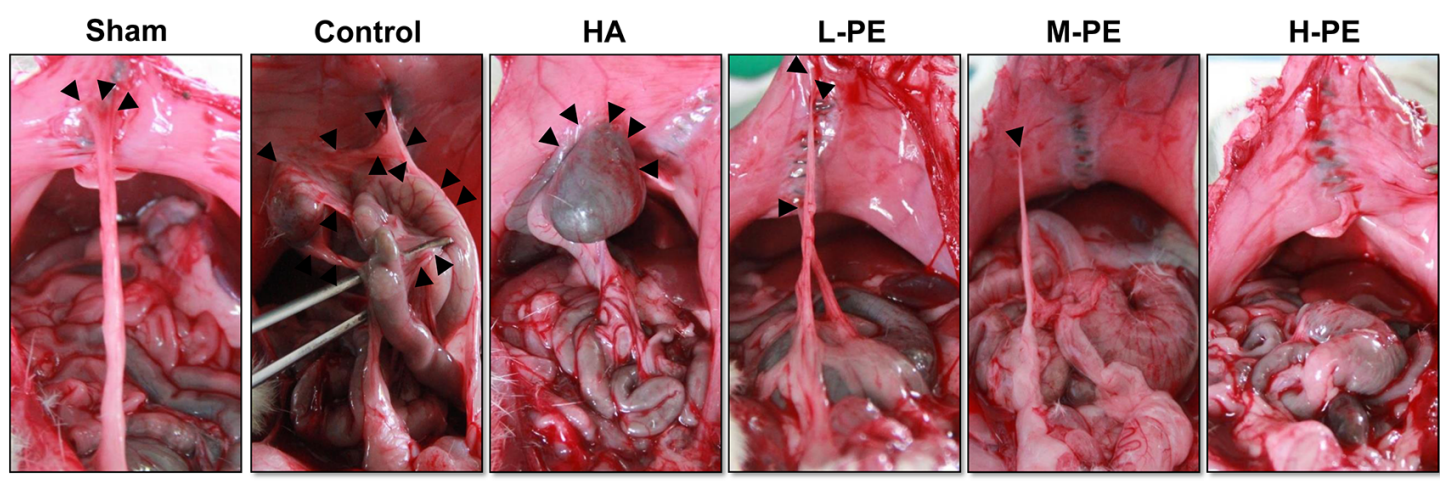

B

C
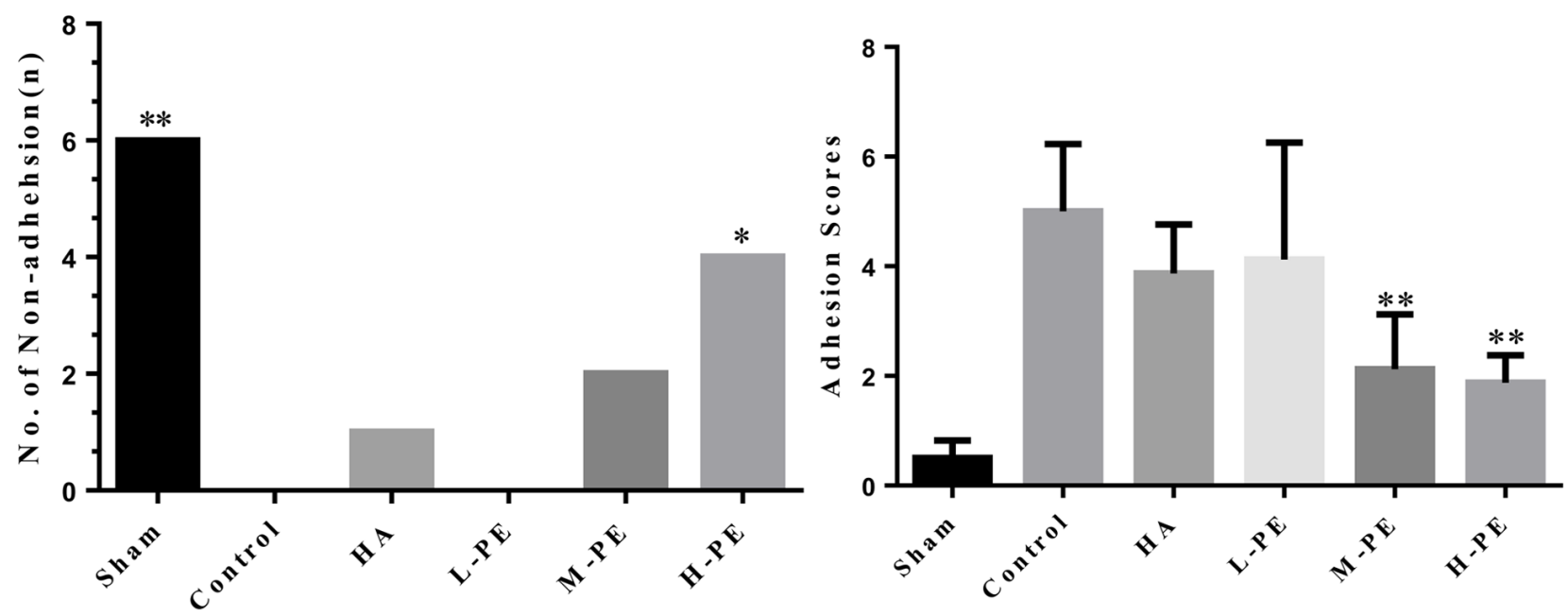

Figure 1: The administration of different concentrations of PE and sodium HA prevented postoperative abdominal adhesion formation in this rat model. (A) The most representative images that showed the postoperative peritoneal adhesions (black triangle) in each groups of rats were chosen. In the sham operation group, almost no adhesions or only a small amount of peritoneal adhesions was present in response to the abdominal incision. In the control group, large adhesions formed in the abdominal cavity, and the adhesion tissue was strong and difficult to separate. In the HA group, moderate abdominal adhesion severity was observed, and the adhesion area was larger than that of the control group. The less significant adhesions in the L-PE group of rats were similar to those in the HA group in magnitude. There were only relatively soft adhesions in some rats in the M-PE and H-PE groups. The number of non-adhesions in each group (B). The adhesion score of the groups (C). ${ }^{*}$ Relative to the control group, $P<0.05$ and ${ }^{* *} P<0.001$. 
expression by immunohistochemical staining (Figure 7A) and western blotting (Figure 4). The result showed that MMP-9 expression in the H-PE group was the highest. The expression of MMP-9 was associated with increasing doses of the drug $(P<0.05)$ (Figure 7C). It was difficult to distinguish the difference between the HA and L-PE groups, and the sham operation group exhibited the lowest expression level. Therefore, these results indicate that PE treatment can reduce collagen deposition in damaged tissue and adhesion formation in the rat model.

\section{PE promotes mesothelial cell repair in the damaged peritoneum in the rat model}

Mesothelial cell continuity and the repair of the cell layer of the damaged rat peritoneum were further evaluated. The expression of cytokeratin was detected via immunohistochemical staining (Figure 7B). In the sham group, the results showed a complete and continuous cell layer on the surface of the two-layer peritoneum (parietal and visceral). In contrast, relatively few mesothelial cells were present on the surface of the damaged two-layer peritoneum in the control group. In the HA and L-PE group, mesothelial cells were equivalent and inconspicuous. Finally, an abundant and a well-repaired mesothelial layer was apparent in the M-PE and H-PE groups $(P<0.05)$ (Figure 7D). In the H-PE group, mesothelial cell repair was more complete and continuous than in the M-PE group. Thus, in our research, PE treatment appeared to contribute to peritoneal mesothelial cellular repair.

A
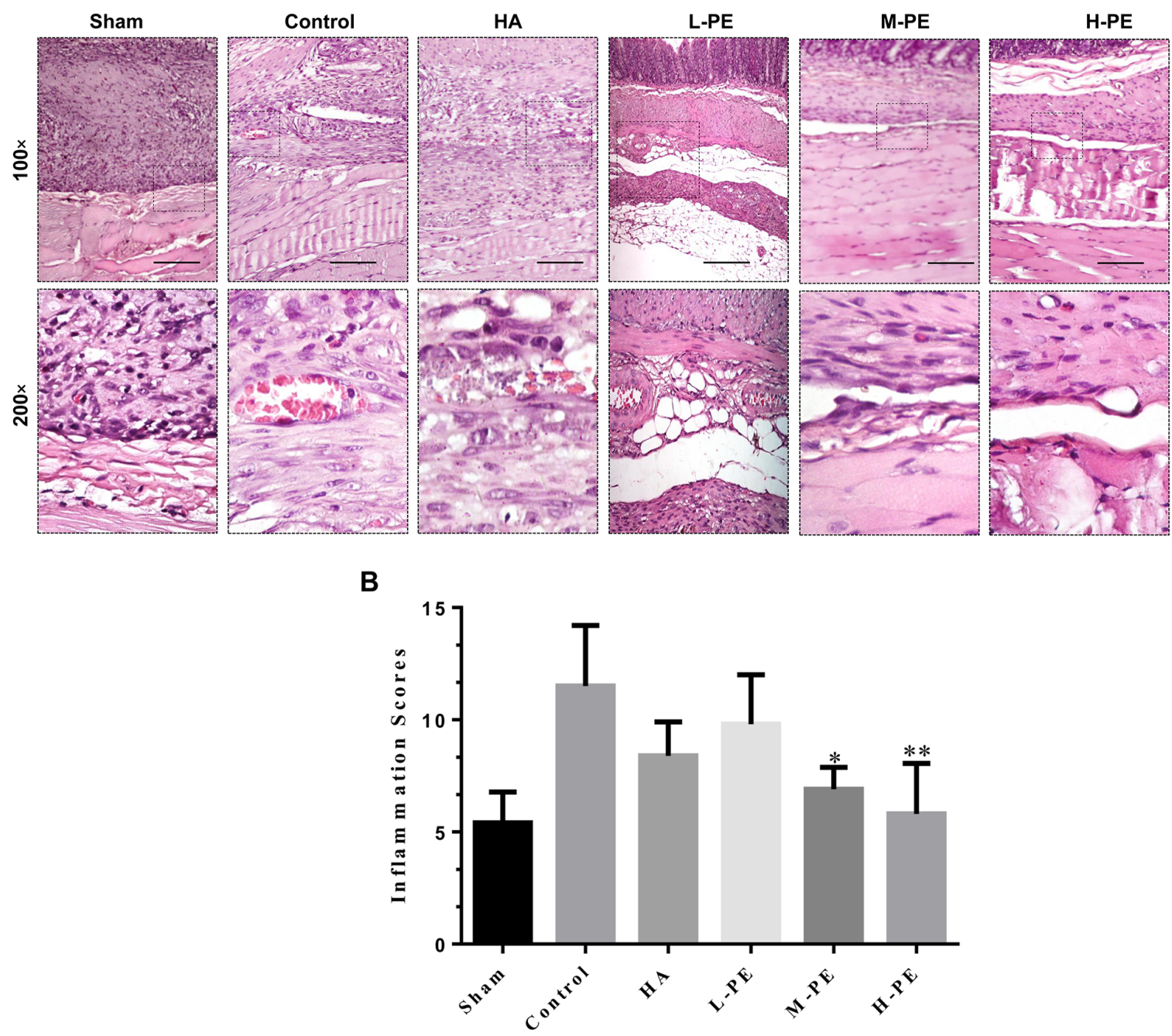

Figure 2: Inflammatory cell infiltration in postoperative peritoneal adhesions or the damaged areas on the opposing parietal peritoneum in each group of rats $(\mathbf{n}=\mathbf{8})$. (A) Representative images of HE staining in the postoperative peritoneal adhesion tissue or the damaged areas on the opposing parietal peritoneum in each group of rats (100× magnification in the upper row, 200× magnification in the lower row; scale bar represents $100 \mu \mathrm{m}$ ). (B) Inflammatory cell infiltration scores for the intra-abdominal adhesive tissue in each group of rats. ${ }^{*}$ Relative to the control group, $P<0.05$ and ${ }^{* *} P<0.001$. 


\section{DISCUSSION}

In the present study, we investigated the antioxidative stress and anti-inflammatory effects of PE on postoperative peritoneal adhesions in an experimental rat model. Our research indicates that PE offers significant protective effects against postoperative peritoneal adhesions through the reduction of inflammation [23] and oxidative stress [24]. Therefore, PE may be useful in the prevention and treatment of peritoneal adhesions.

To date, the most widely accepted method for the prevention of postoperative peritoneal adhesion is to place absorbable barrier or gel between the injured peritoneal surfaces. Studies have shown that HA, chitosan and polyethylene glycol/polylactic acid films

A
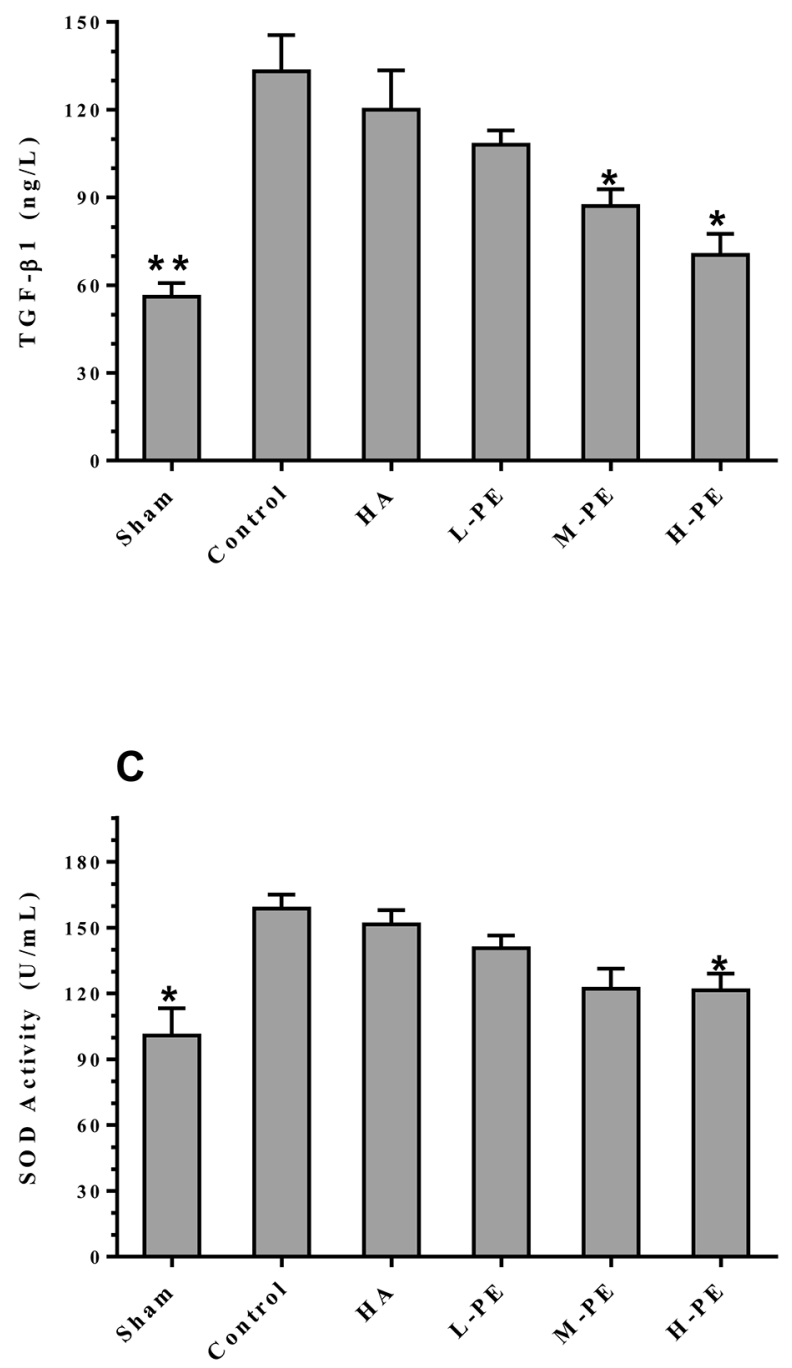

reduce adhesions [25-27]. Currently, HA is an antiadhesion agent that is widely used in clinical practice [28]. However, the anti-adhesion effect of HA is far from satisfactory. Therefore, the effectiveness of PE on preventing adhesion was compared to that of HA in our study.

The generation and elimination of ROS are in a state of dynamic homeostasis when the body is in under normal conditions. The oxidant and anti-oxidant imbalance plays a critical role in pulmonary fibrosis models and fibrotic diseases [29]. ROS is an oxidant that can activate genes that regulate fibroblast proliferation and cell fate [30] and thereby promote peritoneal adhesion fibrosis. Under disease or injury conditions, homeostasis is disrupted, and the anti-oxidant system is disabled due to tissue

\section{B}
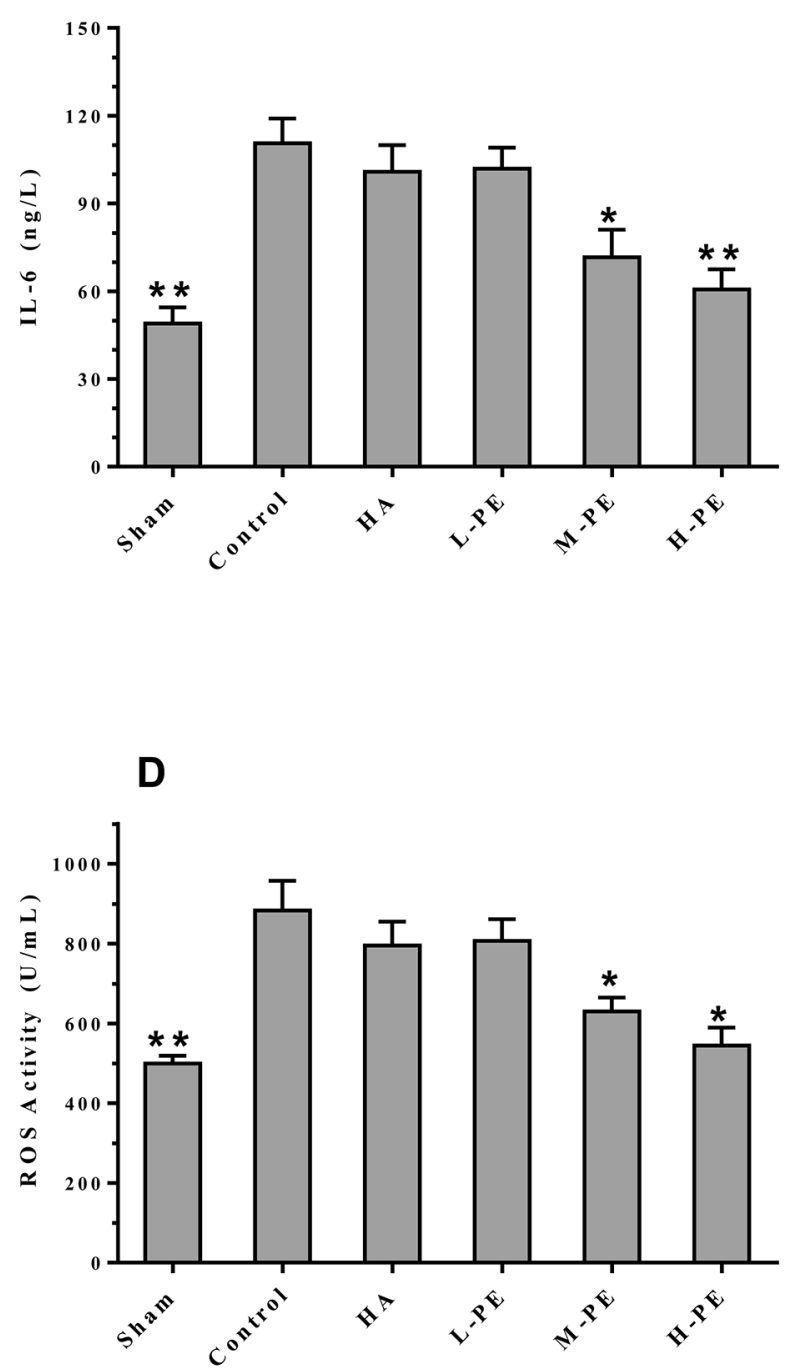

Figure 3: Inflammatory factors from arterial blood were quantified by ELISA. The values (ANOVA) in the L-PE and M-PE groups were compared with the values in the control group (A and B). The abdominal exudatives of SOD in the H-PE group (C) and the ROS in the M-PE and H-PE groups (D) were significantly lower than in the control group. ${ }^{*}$ Relative to the control group, $P<0.05$, ${ }^{* *} P<0.001$. 
damage [31]. Oxidative stress stimulates the formation of peritoneal adhesion, and it is propitious to prevent the formation of adhesion by inhibiting the oxidative stress pathway [32]. in vitro and in vivo, $\mathrm{PE}$ protects against rheumatoid arthritis and cellular apoptosis via the suppression of oxidative stress [33]. In the present rat model study, treatment with PE decreased ROS and SOD activity in the enterocoelia and increased the expression of SOD in peritoneal adhesion specimens. Therefore, we suggest that PE treatment can prevent the development of postoperative peritoneal adhesions and that this therapeutic effect of PE is due to an elevation in the levels of locoregional SOD.

When the peritoneum is damaged, multiple cytokines are secreted [34], among which TGF- $\beta 1$ and
IL-6 are deemed to be extremely important for fibrogenesis [35]. Therefore, inflammation may augment TGF- $\beta 1$ and IL-6, promoting the formation of peritoneal adhesion. An increase in TGF- $\beta 1$ gene expression has been detected in inflamed, damaged arthritis tissue [36]. TGF- $\beta 1$ is also regarded as the main fibrogenic and collagenic component in the inflammatory macrophages that infiltrate damaged tissue [37]. IL-6 is a profibrotic cytokine produced by $\mathrm{B}$ cells. In damaged tissue, IL- 6 promotes fibrosis via its ability to induce fibroblast proliferation, alter tissue inhibitor of metalloproteinase (TIMP) and MMP expression and increase collagen deposition [38]. It has been reported that local alterations of MMPs and TIMPs are regulated by TGF- $\beta 1$ [39] and hypoxia in mesothelial cells. Of the MMPs, MMP-9 is regarded as a marker of

A

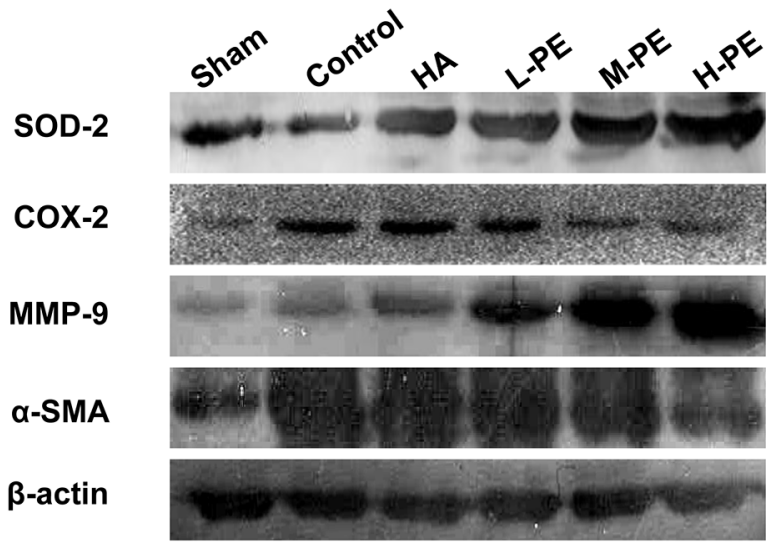

B
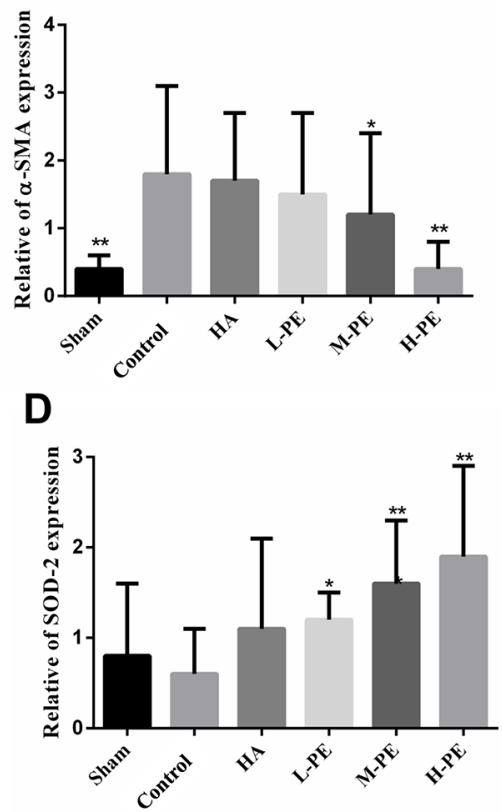

C
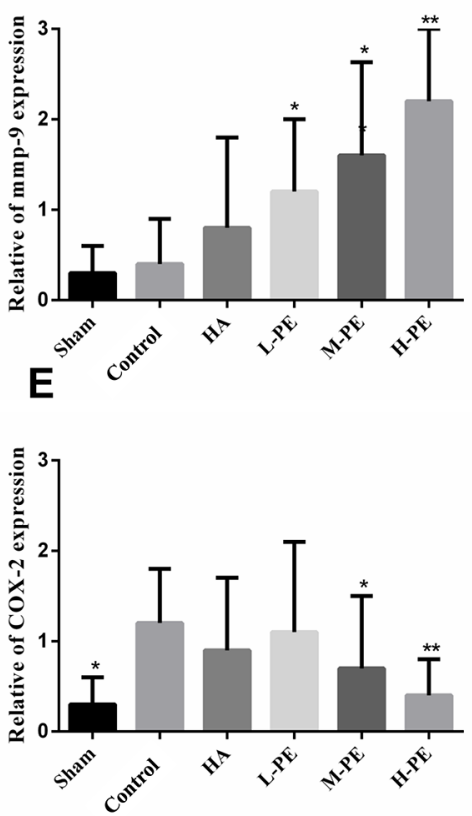

Figure 4: Expression of phosphorylated SOD-2, COX-2, MMP-9 and $\alpha$-SMA proteins in the peritoneal adhesions or the damaged areas on the opposing parietal peritoneum was detected by western blotting (A). The relative expression of $\alpha$-SMA, MMP-9, COX-2 and SOD-2 in different groups (B, C, D and E). ${ }^{*}$ Relative to the control group, $P<0.05$ and ${ }^{* *} P<0.001$. 
fibrosis; the high expression of MMP-9 corresponds to low fibrosis and the degradation of matrix components. Furthermore, MMP-9 is involved in various aspects of inflammation, including the healing and repairing of tissue injury and in remodeling processes [40]. In the present study, the levels of TGF- $\beta 1$, IL- 6 and inflammatory cell infiltrate were clearly lower in the M-PE and H-PE experimental groups than in the sham and control groups. Immunohistochemical and western blotting detection revealed the lower expression of collagen I and $\alpha$-SMA and the higher expression of MMP-9 in the PE groups than in the control group. We therefore conclude that $\mathrm{PE}$ suppresses postoperative peritoneal adhesions by reducing TGF- $\beta 1$ and IL- 6 levels and the associated inflammatory reaction.

In our study, we also investigated $\mathrm{COX}-2$, which has proinflammatory activities. In previous studies [41, 42], the application of a COX-2 inhibitor to arthritic rat models quickly ameliorated paw edema and relieved joint inflammation. In animal models of arthritis, the selective inhibition of COX-2 activity also modulates systemic and local cytokine generation. Moreover, a previous study [15] showed that selective COX-2 inhibitors can be used to prevent intra-abdominal adhesion
A
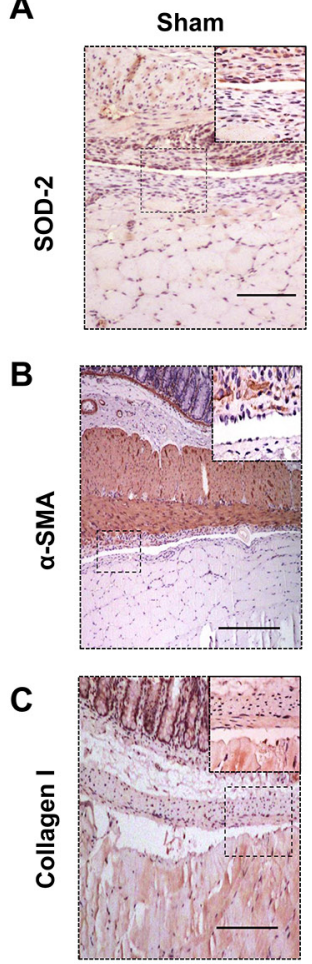

D

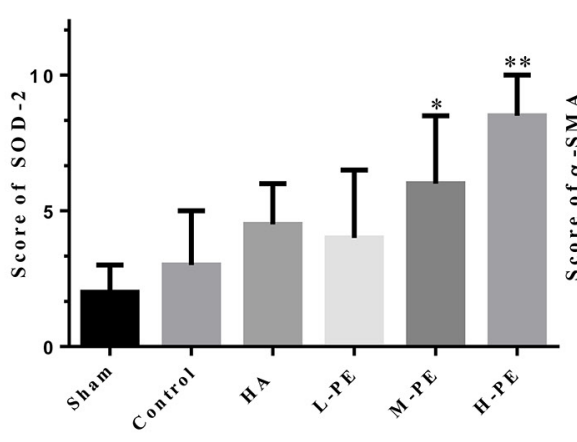

Control
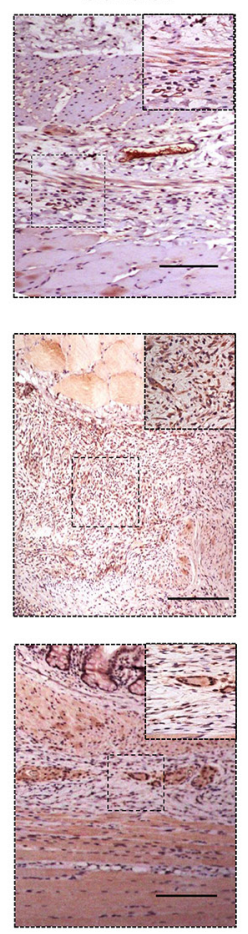
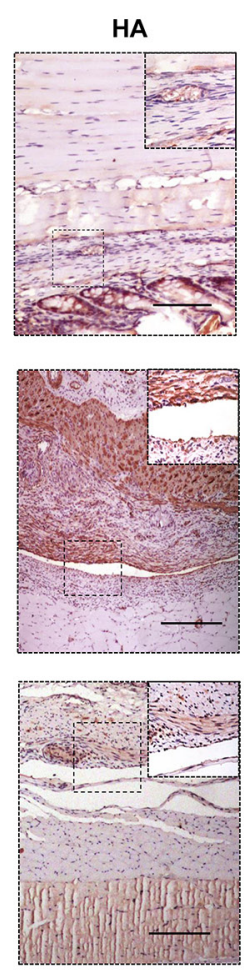

$\mathbf{E}$
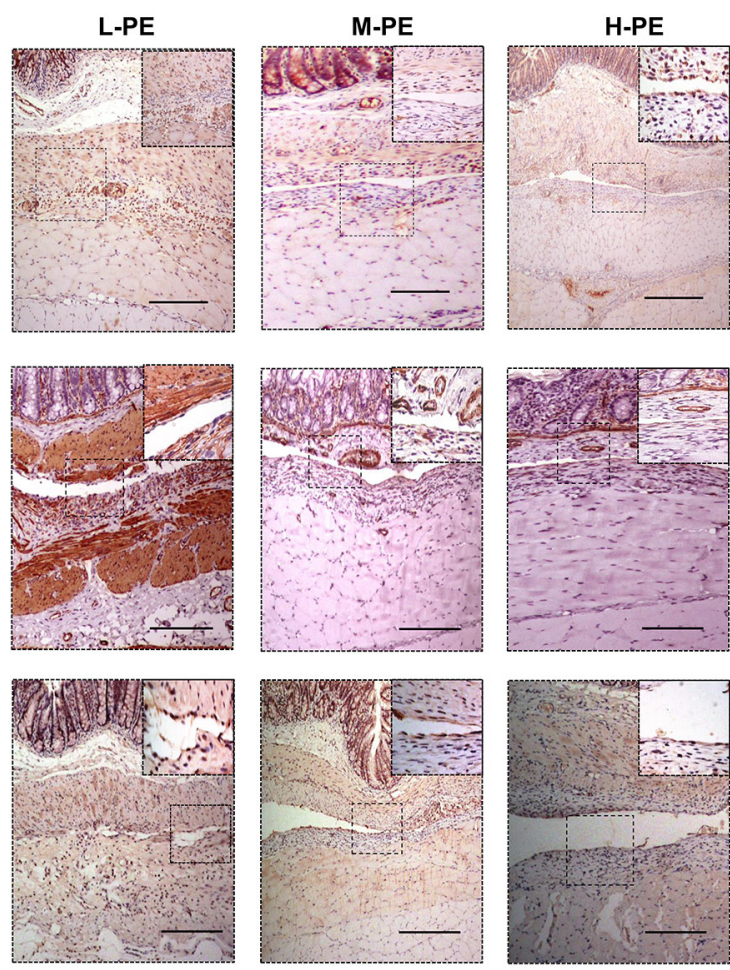

$\mathbf{F}$

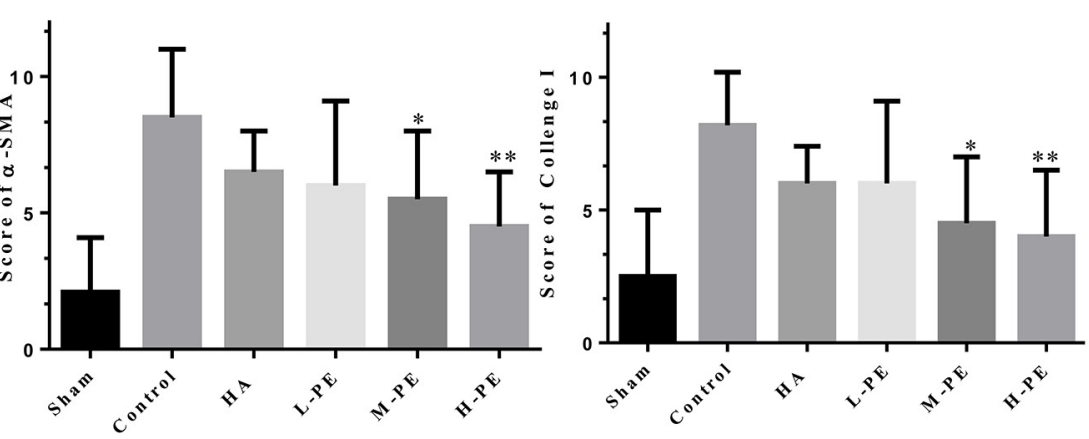

Figure 5: Immunohistochemical staining of SOD-2, $\alpha$-SMA and collagen $I$ in the postoperative peritoneal adhesions or the damaged areas on the opposing parietal peritoneum in each group of rats $(100 \times$ magnification in the upper row, $200 \times$ magnification in the upper left corner). Relative to the sham group, the severity of adhesions was lower, and the higher the SOD-2 expression, the stronger the anti-oxidative stress activity (A). With the increase in drug concentration, $\alpha$-SMA (B) and collagen I (C) staining was gradually reduced, and the formation of fibers was gradually decreased. The black scale bar represents $100 \mu \mathrm{m}$. (D, E and F) show the SOD-2, $\alpha$-SMA and collagen I staining scores in the different groups. ${ }^{*}$ Relative to the control group, $P<0.05$ and ${ }^{* *} P<0.001$. 
formation in rat models. IL- 6 is one of the cytokines that promotes the development of arthritis. in vitro and in vivo, systemic IL-6 was spontaneously produced in controls; its expression was rapidly reversed when COX2 activity was inhibited. Furthermore, the inhibition could markedly reduce inflammatory cell infiltration and the inflammation of arthritis tissue [43]. This result is consistent with the outcomes of previous studies [23, 24], which have shown that PE suppresses arthritis and hepatic reperfusion injury by decreasing $\mathrm{COX}-2$ expression in rat models. Consequently, COX-2 likely contributes to peritoneal adhesion by potentiating the depletion of IL6 . In our results, the expression of IL-6 and COX-2 and the cellular infiltrate in the H-PE group were significantly decreased. The extent of peritoneal adhesions was also less than that of other groups. The inhibition of COX-2 by $\mathrm{PE}$ to partially decrease inflammatory cell infiltrates in peritoneal adhesion specimens likely explains the reduction in the levels of IL- 6 and COX-2 proteins.

This study provided evidence for the anti-adhesion effects from the systematic function of PE and for the Enhanced Recovery After Surgery $\left(\mathrm{ERAS}^{\circledR}\right)$ concept (colonic surgery) in which patients are encouraged to consume moderate amounts of water and food as soon as possible after surgery. The early water and food intake could promote intestinal motility [44] without any complications [45]. Therefore, we believe that it is safe and feasible to take PE as an anti-adhesion agent via gavage.

This study provides a reference for the clinical treatment of peritoneal adhesions, but it cannot be considered absolutely conclusive because the human body is often compromised by a variety of diseases, the internal environment is more complex than that of the mouse, and there are also individual variations. Additional clinical trials and the elucidation of underlying molecular mechanisms are required to verify the effects of PE on adhesion formation.

We conclude that PE markedly suppressed postoperative peritoneal adhesions in a dose-dependent manner in a rat model. The protective effects of PE in adhesion treatment may be due to anti-oxidative stress and anti-inflammatory mechanisms and the inhibition of COX-2. Accordingly, we suggest that PE could be used
A

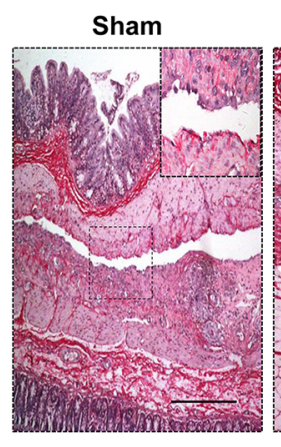

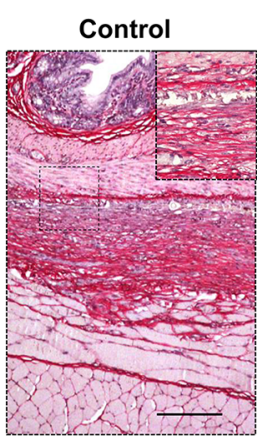
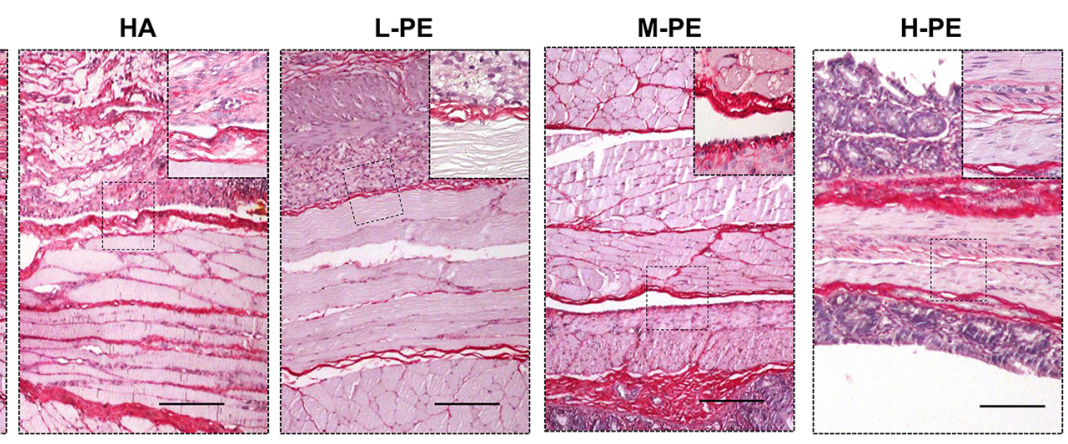

B

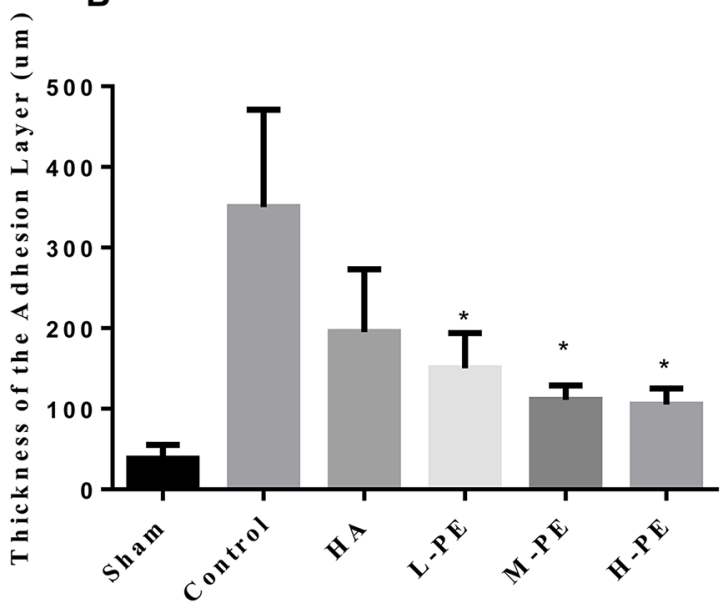

Figure 6: Picrosirius red staining revealed the presence of fibrosis in the postoperative peritoneal adhesions or in the damaged areas on the opposing parietal peritoneum in each group of rats $(\mathbf{n}=\mathbf{8})$. (A) Representative images of picrosirius red staining (100×; insets, 200×) in each group. (B) The collagen deposit thickness in the adhesive tissue of each group of rats was reduced relative to the control group. ${ }^{*}$ Relative to the control, $P<0.05$. 
as a therapeutic agent for the inhibition of postoperative peritoneal adhesions. However, more in-depth studies are needed before PE can be applied in the prevention of postoperative peritoneal adhesions in patients.

A
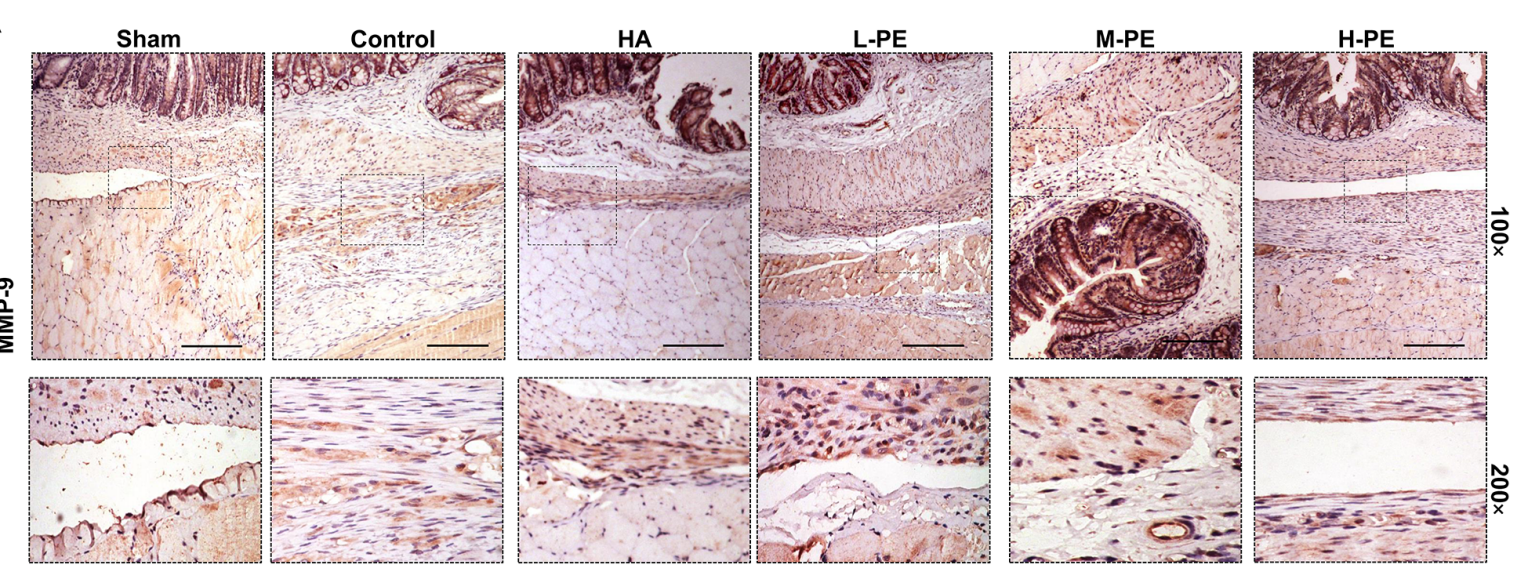

B
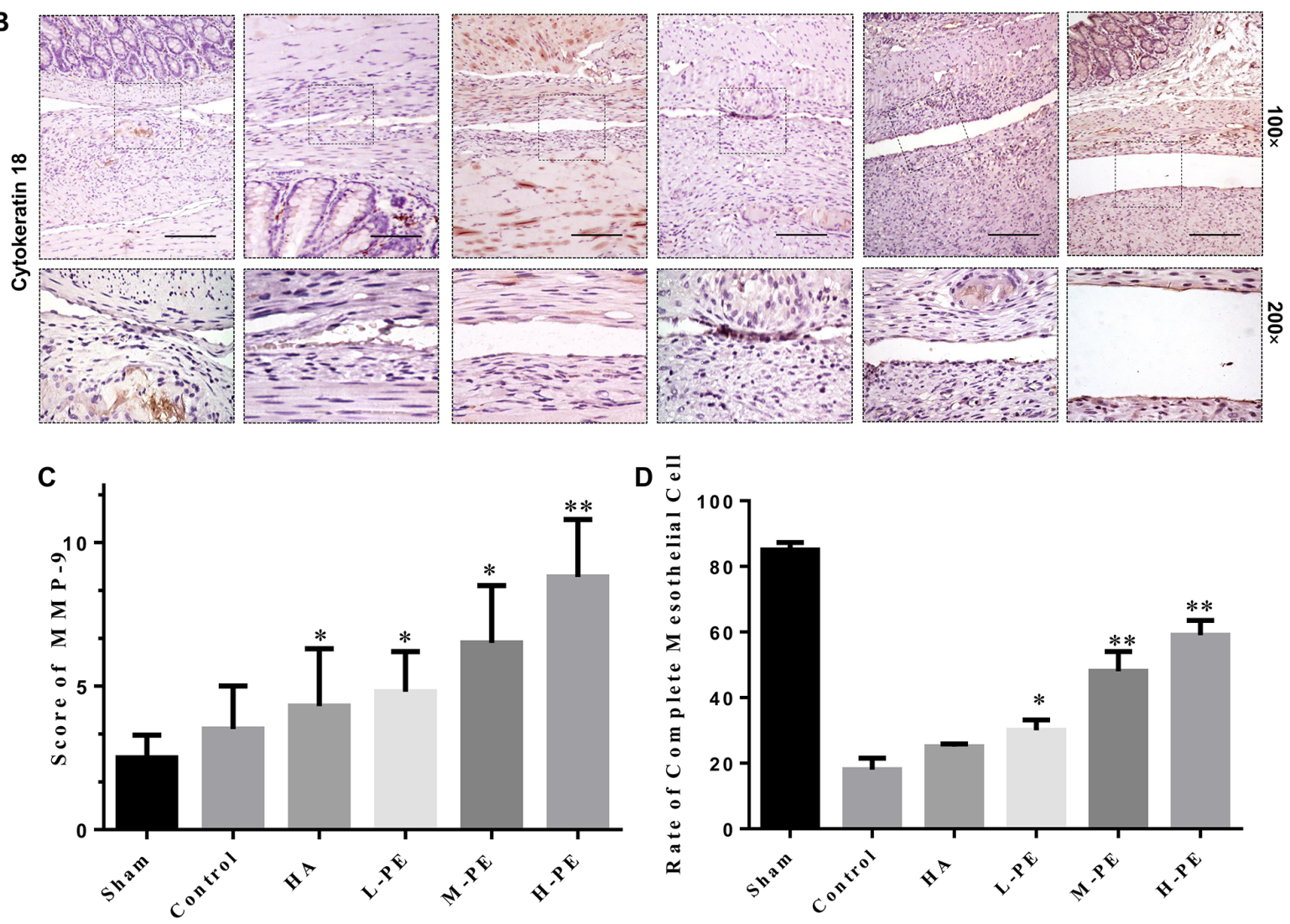

Figure 7: Immunohistochemical staining of MMP-9 and cytokeratin in the postoperative peritoneal adhesions or the damaged areas on the opposing parietal peritoneum in each group of rats $(100 \times$ magnification in the upper row, and 200 $\times$ magnification in the bottom row). With the exception of the sham group, the adhesion severity was lower, the MMP-9 expression was higher, and the collagen degradation was improved compared to the control group (A). In (B): in the sham operation group, the epithelial cells were in a complete, continuous line with almost no brown stain, indicating that the mesothelial cell layer was unscathed). In the control group, the brown thin continuous line was completely interrupted; the interrupted brown line indicates an injured mesothelial cell layer. In the HA group, incomplete continuous brown lines were observed, indicating that the peritoneal mesothelial cell layer was not completely repaired by regeneration. In the PE groups, the L-PE performance was similar to that of the HA group, and in the M-PE and H-PE groups, continuous brown lines were observed, indicating that the peritoneal mesothelial cell layer was completely repaired by regeneration. A thin continuous brown line was formed, indicating that the peritoneal mesothelial cell layer was complete. The MMP-9 staining score in the different groups (C). The rate of complete mesothelial cells (D). ${ }^{*}$ Relative to the control group, $P<0.05$ and ${ }^{* *} P<0.001$. 


\section{MATERIALS AND METHODS}

\section{Experimental animals}

Forty-eight Sprague-Dawley male rats (weight, 180200 g) were obtained from the Animal Resource Center of Xi' an Jiaotong University. The rats were kept in cages under standard conditions and were allowed free access to food and water. The guidelines of the Animal Care and Use Committee of the Xi'an Jiaotong University Health Science Center served as the reference standard. Our experimental procedures and processes were approved by the Ethics Committee.

\section{Model establishment}

The rats were randomly divided into six groups as follows. The animals were anesthetized with a $50 \mathrm{mg} / \mathrm{kg}$ peritoneal injection of pentobarbital for all procedures. Prior to incision, the abdomen was shaved, and the skin was sterilized with antiseptics. An approximately $2 \mathrm{~cm}$ incision of the abdominal wall was made for each animal. The anterior cecal surface was gently abraded 30 times to the same extent with a wet swab until partial petechial hemorrhages were generated. The abdominal wall that faced the treated cecum was damaged using a medical electric scalpel over an area of approximately $2 \mathrm{~cm}^{2}$. The cecum was then placed back in its original location. In the sham operation group, cecal abrasion was not performed, and the abdominal wall was not damaged. Our adhesion model was similar to the one previously reported [15], which mimics the operative process with fewer complications. In the Hyaluronan (HA) group, 1 $\mathrm{mL}$ of HA gel was applied on two sides of the trauma and its surrounding area. The three PE groups received low-dose PE (dissolved in normal saline; purity $>98 \%$, Sigma-Aldrich Co. LLC., St. Louis, MO, USA) at $10 \mathrm{mg} /$ $\mathrm{kg}$ (L-PE); moderate-dose PE at $20 \mathrm{mg} / \mathrm{kg}$ (M-PE) or highdose PE at $40 \mathrm{mg} / \mathrm{kg}$ (H-PE) via gavage for 7 days. The sham, control and HA groups were given the same amount of normal saline by gavage for 7 days. The abdominal incision was closed in two layers with a continuous $3 / 0$ silk suture.

\section{Measurements of the grades of adhesions}

After 7 days, a U-shaped incision was made to estimate the adhesions. Subsequently, two independent observers scored adhesion formation in each group of rats blindly in triplicate. The adhesion scoring systems were applied to evaluate both the degree of severity [46] and the extent $[47,48]$ of adhesion (Supplementary Tables 1 and 2). The overall adhesion score for each animal was the total of the extent score and the severity score. The treatment groups were compared with the control group, and $P<0.05$ indicated a statistically significant difference. After the adhesions were scored, tissue specimens (adhesion tissue, injured cecum wall and parietal peritoneum) and arterial blood samples were collected for the following analyses.

\section{Histological study}

Hematoxylin and eosin (HE) staining was performed for the microscopic histological grading of inflammation. The descriptive system of histology was used to grade the healing process, inflammatory cell reaction, fibroblast activity and neocapillary formation. The system standard [49] is presented in Supplementary Table 3.

\section{Immunohistochemical analysis}

The specimens were removed from $-80^{\circ} \mathrm{C}$ conditions, embedded in paraffin, and cut into 5 consecutive $4-\mu \mathrm{m}$ thick sections. After deparaffinization in an oven at $60^{\circ} \mathrm{C}$ for $3 \mathrm{~h}$, the sections were rehydrated with 2 and 3 changes of xylene and ethanol, respectively. A microwave was used for antigen retrieval. The endogenous peroxide activity was quenched with $3 \%$ hydrogen peroxide for $20 \mathrm{~min}$ at room temperature, and the sections were then rinsed with flowing water 3 times for 5 min per rinse. Nonspecific binding was blocked by BCA (Maixin Biotech. Co., Ltd, Fuzhou, China) goat serum for $20 \mathrm{~min}$ at room temperature according to the manufacturer's instructions. Sections were incubated with rat monoclonal antibodies that targeted collagen-1 (SAB4200678, Sigma, Chicago, USA, 1:200 dilution), superoxide dismutase-2 (SOD-2, SAB2702311, Abcam,Cambridge, UK, 1:200 dilution), cytokeratin 18 (ab27553, Abcam, Cambridge, UK, 1:300 dilution), and $\alpha$-SMA (sc-53015, Santa Cruz Biotechnology, 1:300 dilution) at $4^{\circ} \mathrm{C}$ overnight. Then, the sections were incubated in secondary anti-rat antibodies (Santa Cruz Biotechnology, Inc.) for $35 \mathrm{~min}$ at $37^{\circ} \mathrm{C}$. After washing with PBS, the sections were stained using a DAB Substrate Kit (Thermo Fisher Scientific, Waltham, USA) to visualize the antigen-antibody complex.

To evaluate the staining index, a minimum of five randomly chosen high-power fields were inspected for each section using the scoring system (Supplementary Table 4). The total staining scores (0-12) were determined by multiplying by the score for staining intensity with the score for positive area. The cytokeratin level was evaluated by the average rate of complete and stained mesothelial cells in the selected fields.

\section{Western blotting analysis}

After treatment for 7 days, $20 \mathrm{mg}$ specimens were obtained and incubated on ice with $150 \mu \mathrm{l}$ tissue lysis buffer (Thermo Fisher Scientific). After $1 \mathrm{~h}$, the homogenate was centrifuged at $800 \times \mathrm{g}$ for $15 \mathrm{~min}$ at $4^{\circ} \mathrm{C}$, and the supernatant protein concentration was determined via BAC methods with a protein extraction kit (Fermentas, Beijing, China). We loaded uniform samples of $40 \mu \mathrm{g}$ of protein onto the sodium dodecyl sulfate-polyacrylamide gels and 
transferred gels to polyvinylidene fluoride membranes (Millipore, Billerica, MA, USA). The following antibodies were used for Western blotting analysis in our study: anti-COX-2 (Santa Cruz Biotechnology Inc., Dallas, TX, USA; 1:800 dilution), anti-SOD-2 (SAB2702311, Abcam, Cambridge, UK, 1:500 dilution), anti- $\alpha$-SMA (sc-53015, Santa Cruz Biotechnology, 1:1,000 dilution), anti-MMP-9 (SAB5300247, Sigma, Chicago, USA, 1:800 dilution), and anti- $\beta$-actin (Santa Cruz Biotechnology, 1:5,000 dilution). The samples were incubated for $12 \mathrm{~h}$ at $4^{\circ} \mathrm{C}$. Membranes were incubated with the secondary rat antibodies at $37^{\circ} \mathrm{C}$ for $2 \mathrm{~h}$. Band intensities were measured with Image-Pro Plus software (v. 5.0; Media Cybernetics, Inc., Rockville, MD, USA).

\section{Collagen deposition detected via picrosirius red staining}

A $0.1 \%$ picrosirius red solution (Direct Red 80; Sigma-Aldrich Co) was used to stain collagen. Weigert's hematoxylin (Wako Pure Chemical Industries, Ltd., Osaka, Japan) was applied as a counterstain. The number of positively stained areas was detected via the microscope (Olympus, MD, Japan) in eight randomly selected fields, and the mean of the eight values was considered the collagen thickness in the adhesions.

\section{ELISA analysis}

Arterial blood was collected into evacuated tubes, and coagulation was promoted. The tubes were then centrifuged at $13,000 \times \mathrm{g}$ for $15 \mathrm{~min}$ at $4^{\circ} \mathrm{C}$, and the supernatant was preserved in a $-80^{\circ} \mathrm{C}$ refrigerator for biochemical analysis. The levels of transforming growth factor- $\beta 1$ (TGF- $\beta 1)$ and IL-6 in serum were tested with commercial ELISA kits (Huamei Biological Technology Co., Ltd., Wuhan, PRC eBioscience) according to the instruction manuals.

\section{SOD activity and ROS measurement}

Enterocoelic fluid specimens were acquired from the abdomens of each animal and then centrifuged at $3,000 \times \mathrm{g}$ for $30 \mathrm{~min}$ [50]. The supernatant was stored at $-20^{\circ} \mathrm{C}$ for tests. The levels of SOD (A001; Nanjing Jiancheng Bioengineering Institute, Nanjing, China) and ROS (E004; Nanjing Jiancheng Bioengineering Institute, Nanjing, China) were measured. The assay was conducted according to the manufacturer's instructions.

\section{Statistical analysis}

Statistical analyses were performed using SPSS 18.0 (SPSS Inc., Chicago, IL). Values are described as the mean \pm standard deviation. The Mann-Whitney U analysis was used to compare adhesion formation in the two groups. The mean values of groups were analyzed via one-way analysis of variance (ANOVA). All differences were considered to be statistically significant at a $P$ value of $<0.05$.

\section{Author contributions}

Xuqi Li designed the study. Qi Gao, Guangbing Wei, Yunhua Wu, Na Yao, Cancan Zhou and Kai Wang performed the experiments. Qi Gao and Guangbing Wei performed statistical analysis. Kang Wang, Xuejun Sun and Xuqi Li analyzed the results. Qi Gao and Xuqi Li wrote the manuscript.

\section{CONFLICTS OF INTEREST}

The authors declare that they have no conflicts of interest to disclose.

\section{FUNDING}

This study was supported by the National Natural Science Foundation of China (No. 81572734), the Scientific and Technological Development Research Project Foundation by Shaanxi Province (2016SF-121) and the Fundamental Research Funds for the Central Universities in Xi'an Jiaotong University.

\section{REFERENCES}

1. Ellis H, Moran BJ, Thompson JN, Parker MC, Wilson MS, Menzies D, McGuire A, Lower AM, Hawthorn RJ, O'Brien F, Buchan S, Crowe AM. Adhesion-related hospital readmissions after abdominal and pelvic surgery: a retrospective cohort study. Lancet. 1999; 353: 1476-80.

2. Lower AM, Hawthorn RJ, Clark D, Boyd JH, Finlayson AR, Knight AD, Crowe AM; Surgical and Clinical Research (SCAR) Group. Adhesion-related readmissions following gynaecological laparoscopy or laparotomy in Scotland: an epidemiological study of 24046 patients. Hum Reprod. 2004; 19: 1877-85.

3. Ellis H, Crowe A. Medico-legal consequences of post-operative intra-abdominal adhesions. Int J Surg. 2009; 7: 187-91.

4. Rajab TK, Wallwiener M, Talukdar S, Kraemer B. Adhesion-related complications are common, but rarely discussed in preoperative consent: a multicenter study. World J Surg. 2009; 33: 748-50.

5. Lower AM, Hawthorn RJ, Ellis H, O'Brien F, Buchan $\mathrm{S}$, Crowe AM. The impact of adhesions on hospital readmissions over ten years after 8849 open gynaecological operations: an assessment from the Surgical and Clinical Adhesions Research Study. BJOG. 2000; 107: 855-62.

6. Wilson MS. Practicalities and costs of adhesions. Colorectal Dis. 2007; 9: 60-5.

7. Karimi M, Yazdan AS, Parsaei P, Rafieian-Kopaei M, Ghaheri H, Ezzati S. The effect of ethanol extract of rose 
(Rosa damascena) on intra-abdominal adhesions after laparotomy in rats. Wounds. 2016; 28: 167-74.

8. Wei G, Chen X, Wang G, Fan L, Wang K, Li X. Effect of resveratrol on the prevention of intra-abdominal adhesion formation in a rat model. Cell Physiol Biochem. 2016; 39: $33-46$.

9. Zhou C, Jia P, Jiang Z, Chen K, Wang G, Wang K, Wei G, $\mathrm{Li}$ X. Preventive effects of the intestine function recovery decoction, a traditional Chinese medicine, on postoperative intra-abdominal adhesion formation in a rat model. Evid Based Complement Alternat Med. 2016; 2016: 1621894.

10. Wei G, Zhou C, Wang G, Fan L, Wang K, Li X. Keratinocyte growth factor combined with a sodium hyaluronate gel inhibits postoperative intra-abdominal adhesions. Int J Mol Sci. 2016; 17: E1611.

11. Kumar S, Wong PF, Leaper DJ. Intra-peritoneal prophylactic agents for preventing adhesions and adhesive intestinal obstruction after non-gynaecological abdominal surgery. Cochrane Database Syst Rev. 2009; 1: CD005080.

12. Augustin F, Maier HT, Weissenbacher A, Ng C, Lucciarini P, Öfner D, Ulmer H, Schmid T. Causes, predictors and consequences of conversion from VATS to open lung lobectomy. Surg Endosc. 2016; 30: 2415-21.

13. Diamond MP. Reduction of postoperative adhesion development. Fertil Steril. 2016; 106: 994-7. e1.

14. Donnez J, Binda MM, Donnez O, Dolmans MM. Oxidative stress in the pelvic cavity and its role in the pathogenesis of endometriosis. Fertil Steril. 2016; 106: 1011-7.

15. Wei G, Chen X, Wang G, Jia P, Xu Q, Ping G, Wang K, Li $\mathrm{X}$. Inhibition of cyclooxygenase-2 prevents intra-abdominal adhesions by decreasing activity of peritoneal fibroblasts. Drug Des Devel Ther. 2015; 9: 3083-98.

16. Koninckx PR, Gomel V, Ussia A, Adamyan L. Role of the peritoneal cavity in the prevention of postoperative adhesions, pain, and fatigue. Fertil Steril. 2016; 106: 998-1010.

17. Binnebösel M, Rosch R, Junge K, Lynen-Jansen $P$, Schumpelick V, Klinge U. Macrophage and T-lymphocyte infiltrates in human peritoneal adhesions indicate a chronic inflammatory disease. World J Surg. 2008; 32: 296-304.

18. Ohashi K, Yoshimoto T, Kosaka H, Hirano T, Iimuro Y, Nakanishi K, Fujimoto J. Interferon $\gamma$ and plasminogen activator inhibitor 1 regulate adhesion formation after partial hepatectomy. Br J Surg. 2014; 101: 398-407.

19. Wei Y, La L, Wang L, Batey R, Wang C, Li Y. Paeoniflorin and liquiritin, two major constituents in Chinese herbal formulas used to treat hyperprolactinemia-associated disorders, inhibits prolactin secretion in prolactinoma cells by different mechanisms. J Ethnopharmacol. 2017; 204: 36-44.

20. Jia Z, He J. Paeoniflorin ameliorates rheumatoid arthritis in rat models through oxidative stress, inflammation and cyclooxygenase 2. Exp Ther Med. 2016; 11: 655-9.

21. Ni J, Yang D, Song L, Li C. Protective effects of paeoniflorin on alveolar bone resorption and soft-tissue breakdown in experimental periodontitis. J Periodontal Res. 2016; 51: 257-64.

22. Yu J, Zhu X, Qi X, Che J, Cao B. Paeoniflorin protects human EA. hy926 endothelial cells against gammaradiation induced oxidative injury by activating the NF-E2related factor $2 /$ heme oxygenase-1 pathway. Toxicol Lett. 2013; 218: 224-34.

23. Zheng YQ, Wei W, Zhu L, Liu JX. Effects and mechanisms of Paeoniflorin, a bioactive glucoside from paeony root, on adjuvant arthritis in rats. Inflamm Res. 2007; 56: 182-8.

24. Tao YE, Wen Z, Song Y, Wang H. Paeoniflorin attenuates hepatic ischemia/reperfusion injury via anti-oxidative, antiinflammatory and anti-apoptotic pathways. Exp Ther Med. 2016; 11: 263-8.

25. Hu J, Fan D, Lin X, Wu X, He X, He X, Wu X, Lan P. Safety and efficacy of sodium hyaluronate gel and chitosan in preventing postoperative peristomal adhesions after defunctioning enterostomy: a prospective randomized controlled trials. Medicine (Baltimore). 2015; 94: e2354.

26. Yang B, Gong C, Zhao X, Zhou S, Li Z, Qi X, Zhong Q, Luo F, Qian Z. Preventing postoperative abdominal adhesions in a rat model with PEG-PCL-PEG hydrogel. Int J Nanomedicine. 2012; 7: 547-57.

27. Yuan F, Lin LX, Zhang HH, Huang D, Sun YL. Effect of carbodiimide-derivatized hyaluronic acid gelatin on preventing postsurgical intra-abdominal adhesion formation and promoting healing in a rat model. J Biomed Mater Res A. 2016; 104: 1175-81.

28. Tang CL, Jayne DG, Seow-Choen F, Ng YY, Eu KW, Mustapha N. A randomized controlled trial of $0.5 \%$ ferric hyaluronate gel (Intergel) in the prevention of adhesions following abdominal surgery. Ann Surg. 2006; 243: 449-55.

29. Selman M, King TE, Pardo A. Idiopathic pulmonary fibrosis: prevailing and evolving hypotheses about its pathogenesis and implications for therapy. Ann Intern Med. 2001; 134: 136-51.

30. Liu G, Cheresh P, Kamp DW. Molecular basis of asbestosinduced lung disease. Annu Rev Pathol. 2013; 8: 161-87.

31. Baldeiras I, Santana I, Proença MT, Garrucho MH, Pascoal R, Rodrigues A, Duro D, Oliveira CR. Peripheral oxidative damage in mild cognitive impairment and mild Alzheimer's disease. J Alzheimers Dis. 2008; 15: 117-28.

32. Swanson PA, Kumar A, Samarin S, Vijay-Kumar M, Kundu K, Murthy N, Hansen J, Nusrat A, Neish AS. Enteric commensal bacteria potentiate epithelial restitution via reactive oxygen species-mediated inactivation of focal adhesion kinase phosphatases. Proc Natl Acad Sci U S A. 2011; 108: 8803-8.

33. Toyokuni S. Molecular mechanisms of oxidative stress-induced carcinogenesis: from epidemiology to oxygenomics. IUBMB Life. 2008; 60: 441-7.

34. Reijnen MM, Bleichrodt RP, van Goor H. Pathophysiology of intra-abdominal adhesion and abscess formation, and the effect of hyaluronan. Br J Surg. 2003; 90: 533-41. 
35. Reed KL, Fruin AB, Bishop-Bartolomei KK, Gower AC, Nicolaou M, Stucchi AF, Leeman SE, Becker JM. Neurokinin-1 receptor and substance P messenger RNA levels increase during intraabdominal adhesion formation. J Surg Res. 2002; 108: 165-72.

36. Cheong YC, Shelton JB, Laird SM, Richmond M, Kudesia G, Li TC, Ledger WL. IL-1, IL-6 and TNF-alpha concentrations in the peritoneal fluid of women with pelvic adhesions. Hum Reprod. 2002; 17: 69-75.

37. Karaca T, Gözalan AU, Yoldaş Ö, Bilgin BÇ, Tezer A. Effects of tamoxifen citrate on postoperative intraabdominal adhesion in a rat model. Int J Surg. 2013; 11: 68-72.

38. Wang C, Yuan J, Wu HX, Chang Y, Wang QT, Wu YJ, Liu LH, Wei W. Paeoniflorin inhibits inflammatory responses in mice with allergic contact dermatitis by regulating the balance between inflammatory and anti-inflammatory cytokines. Inflamm Res. 2013; 62: 1035-44.

39. Hobson KG, DeWing M, Ho HS, Wolfe BM, Cho K, Greenhalgh DG. Expression of transforming growth factor beta1 in patients with and without previous abdominal surgery. Arch Surg. 2003; 138: 1249-52.

40. Brumann M, Kusmenkov T, Ney L, Kanz KG, Leidel BA, Biberthaler P, Mutschler W, Bogner V. Concentration kinetics of serum MMP-9 and TIMP-1 after blunt multiple injuries in the early posttraumatic period. Mediators Inflamm. 2012; 2012: 435463.

41. Houssiau FA, Devogelaer JP, Van Damme J, de Deuxchaisnes CN, Van Snick J. Interleukin-6 in synovial fluid and serum of patients with rheumatoid arthritis and other inflammatory arthritides. Arthritis Rheum. 1988; 31: 784-8.

42. Theisen-Popp P, Pape H, Müller-Peddinghaus R. Interleukin-6 (IL-6) in adjuvant arthritis of rats and its pharmacological modulation. Int $\mathrm{J}$ Immunopharmacol. 1992; 14: 565-71.
43. Anderson GD, Hauser SD, McGarity KL, Bremer ME, Isakson PC, Gregory SA. Selective inhibition of cyclooxygenase (COX)-2 reverses inflammation and expression of COX-2 and interleukin 6 in rat adjuvant arthritis. J Clin Invest. 1996; 97: 2672-9.

44. Zhang Y, Gu F, Wang F, Zhang Y. Effects of early enteral nutrition on the gastrointestinal motility and intestinal mucosal barrier of patients with burn-induced invasive fungal infection. Pak J Med Sci. 2016; 32: 599-603.

45. Gustafsson UO, Scott MJ, Schwenk W, Demartines N, Roulin D, Francis N, McNaught CE, MacFie J, Liberman AS, Soop M, Hill A, Kennedy RH, Lobo DN, et al. Guidelines for perioperative care in elective colonic surgery: Enhanced Recovery After Surgery (ERAS ${ }^{\circledR}$ ) Society recommendations. Clin Nutr. 2012; 31: 783-800.

46. Parker MC, Ellis H, Moran BJ, Thompson JN, Wilson MS, Menzies D, McGuire A, Lower AM, Hawthorn RJ, O'Briena F, Buchan S, Crowe AM. Postoperative adhesions: ten-year follow-up of 12,584 patients undergoing lower abdominal surgery. Dis Colon Rectum. 2001; 44: 822-9; discussion 829-30.

47. Verhofstad MH, Lange WP, van der Laak JA, Verhofstad AA, Hendriks T. Microscopic analysis of anastomotic healing in the intestine of normal and diabetic rats. Dis Colon Rectum. 2001; 44: 423-31.

48. Lundorff $\mathrm{P}$, van Geldorp H, Tronstad SE, Lalos O, Larsson B, Johns DB, diZerega GS. Reduction of post-surgical adhesions with ferric hyaluronate gel: a European study. Hum Reprod. 2001; 16: 1982-8.

49. Saber A. Effect of honey versus intergel in intraperitoneal adhesion prevention and colonic anastomotic healing: a randomized controlled study in rats. Int J Surg. 2010; 8: 121-7.

50. Nagelschmidt M, Saad S. Influence of polyethylene glycol 4000 and dextran 70 on adhesion formation in rats. J Surg Res. 1997; 67: 113-8. 